\title{
Pure Spinal Epidural Cavernous Hemangioma: A Case Report
}

\author{
Woo Jung Lim, Jin Woo Hur, Seong Yeol Ahn, Jong Joo Rhee, Jong Won Lee, Hyun Koo Lee \\ Department of Neurosurgery, Cheongju St. Mary's Hospital, Cheongju, Korea
}

Pure spinal epidural cavernous hemangioma is difficult to differentiate from ruptured disc material and is rarely reported. It is usually reported in thoracic spine. The patient was a 73-year-old man suffering from chronic pain in the L5 dermatome area in his left leg. Computed tomography scans showed a slightly high-signal intensity epidural mass in lateral recess at the L4 vertebral body. This lesion showed hyper-intensity on T2-weighted magnetic resonance imaging (MRI) and enhancement on T1-weighted MRI with gadolinium enhancement. This mass-like lesion was surgically removed and the patient's symptoms improved without complication. Here, we present a case of pure spinal epidural cavernous hemangioma, which should be included in the differential diagnosis of spinal epidural tumors. Early surgical treatment is important.

Key Words: Hemangioma $\cdot$ Cavernous $\cdot$ Epidural space $\cdot$ Intervertebral disc disease $\cdot$ Magnetic resonance imaging

\section{INTRODUCTION}

Extradural cavernous hemangioma are very rare. In most cases, they arise from the vertebral bodies and extend into the extradural space, ${ }^{6,915)}$. Purely extradural cavernous hemangiomas without any vertebral body involvement are extremely rare, and account for only $4 \%$ of all extradural spinal tumors ${ }^{11,15}$. They are most commonly located in the posterior aspect of the thoracic epidural space and are rarely found in the lumbar space ${ }^{6,7,915)}$. Clinically, they can cause symptoms of radiculopathy, which causes misdiagnosis as herniated intervertebral disc disease.

We present a rare case of a pure lumbar epidural cavernous hemangioma at the L4-5 level. This case is reported because of its rarity, the difficulty in making a preoperative diagnosis, and its favorable outcome after surgical treatment.

\section{CASE REPORT}

The patient was a 73-year-old man who had been suffering from left lateral thigh pain for the last 8 months; his symptoms were exacerbated within the past 3 months. He had progressive weakness in left lower extremity. Upon neurological examination, motor power in both lower extremities and deep tendon reflexes were intact. The patient had no past medical

Corresponding author: Jin Woo Hur

Department of Neurosurgery, Cheongju St. Mary's Hospital, 173-19,

Juseong-ro, Cheongwon-gu, Cheongju 28323, Korea

Tel: +82-43-219-8000, Fax: +82-43-291-8112

E-mail: somalh@naver.com history and the laboratory tests were within normal limits except for mild hyperglycemia and the elevation in epinephrine (EPI; platelet function assay).

On the L-spine X-ray, endplate sclerotic change at L4-5-S1 were observed. L-spine computed tomography (CT) showed a ventral epidural mass around the lateral recess at the level of the L4 vertebra body. The mass was rounded in shape without calcification or acute hemorrhage (Fig. 1). From the L-spine magnetic resonance imaging (MRI), an epidural mass was seen as hyperintense on the T2-weighted images (WI) and isointense on T1-WI. Also, there was slightly heterogeneous staining on the gadolinium-enhancement T1-WI. Additionally, it was observed that the spinal cord was displaced to the left due to the mass (Fig. 2).

A L4 total laminectomy was performed and a dark red encapsulated mass with a soft consistency was found while the thecal sac was retracted. The mass was removed by en-bloc resection along with coagulation and no significant bleeding occurred during the operation. Neurological deterioration did not occur during the postoperative period. The patient's left lateral thigh pain improved without any recurrence of symp-

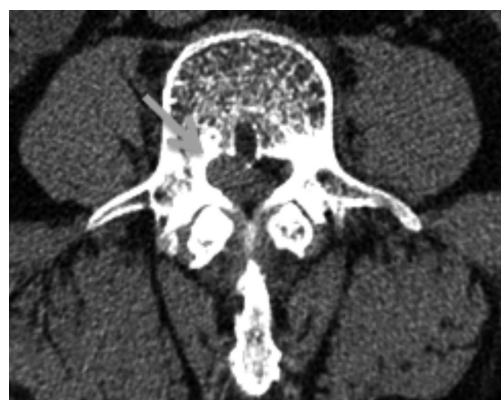

Fig. 1. L-spine computed tomography showing a ventral epidural mass around the lateral recess at the $L 4$ vertebra body level. 


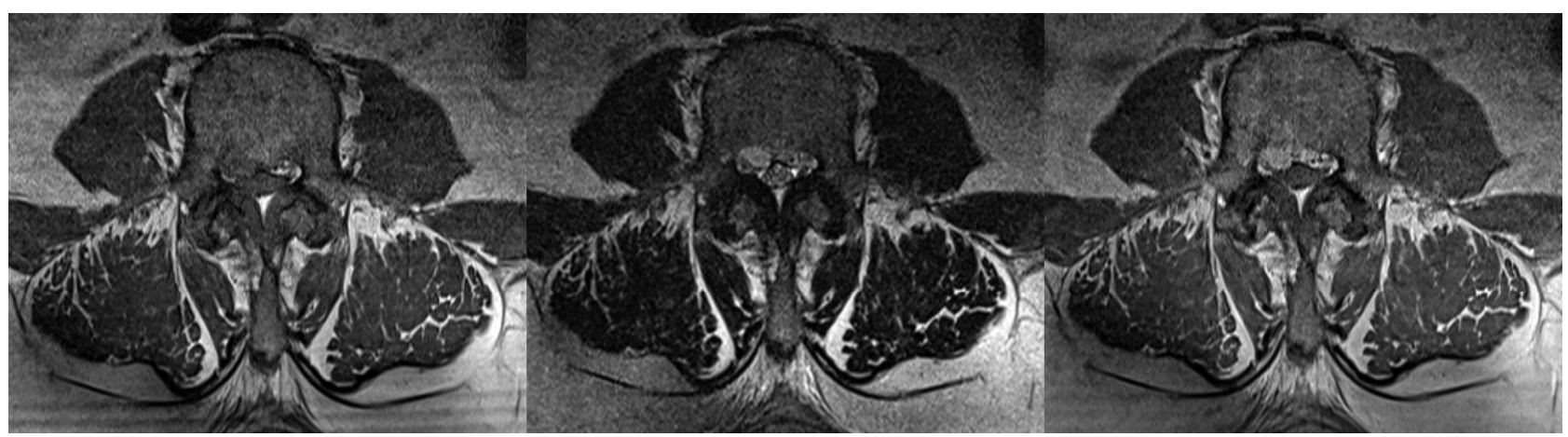

Fig. 2. L-spine magnetic resonance imaging. An epidural mass was seen as isointense on $T 1$-weighted images (Tl-WI) and hyperintense on T2-Wl. Also, there was slight heterogeneous staining on the gadolinium-enhancement T1-WI.

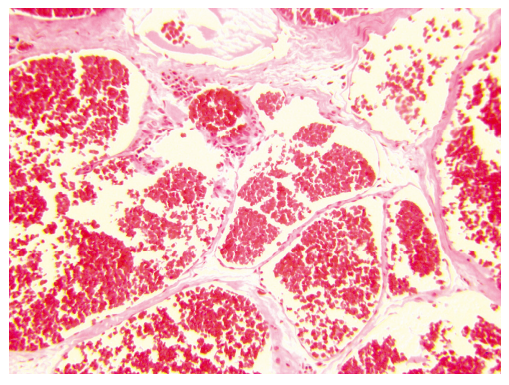

Fig. 3. Microscopic image showing the endothelial lining, which formed a cavernous channel, thus confirming cavernous hemangioma.

toms until the current point, 3 months after the operation.

Histopathological examination revealed approximately 10 $\mathrm{cc}$ of pale pinkish fibrocartilaginous tissue and microscopic imaging allowed observation of the endothelial lining forming a cavernous channel, thus confirming cavernous hemangioma (Fig. 3).

\section{DISCUSSION}

Spinal epidural hemangiomas are rare lesions, constituting approximately $12 \%$ of cases of spinal cord vascular disease ${ }^{2,7}$. A pure epidural cavernous hemangioma was first reported in 1929 by Globus and Doshay. Cavernous hemangiomas are a collection of small capillaries covered with a single layer of endothelium, characterized by lobules, separated by fibrous connective tissue septa, and composed of irregular and dilated vascular channels, ${ }^{4,14}$.

Cavernous hemangiomas are not real neoplasms. Clinical symptoms are caused by the mass effect, vascular movements, hemorrhage, thrombosis, cysts, or cavern formation. Cavernous hemangiomas may occur in any part of the body ${ }^{8}$. They mainly appear in the thoracic spine in an acute manner as the cord or conus are compressed ${ }^{2,3,5,12,14)}$. Occurrence in the lumbar spine is very rare because nerve roots can tolerate compression better than the spinal cord; thus, it presents a chronic clinical course ${ }^{3,7}$. Pain does not resolve spontaneously, unlike in herniated intervertebral disc disease. For this reason, we need to consider cavernous hemangioma in patients suffering from chronic back pain or radicular pain which is not controlled by conservative treatment. If the diagnosis is a spinal cavernous hemangioma, only surgical resection can cure the problem.

As illustrated in our case, a purely epidural hemangioma, although uncommon, should be considered in the differential diagnosis of spinal epidural soft tissue masses. It is very difficult to diagnose before surgery. A study by Tekkök et al. ${ }^{13)}$ reported on 14 surgical resections of cavernous hemangioma, none of which was diagnosed preoperatively.

MRI is the most useful method of diagnosing a cavernous hemangioma. It usually shows an isointense T1-WI and a hyperintense T2-WI. It often shows irregular or lobulated enhancement, and this needs to be differentiated from other diseases like intervertebral disc herniation, schwannoma, neurofibroma, angiolipoma, osteochondroma, synovial cyst, lymphoma, chordoma, and Ewing's sarcoma. For example, intervertebral disc herniation may present with none or peripheral enhancement and schwannoma may present less enhanced or neural foraminal widening. Whereas lymphoma presents as isointense on T2-WI. In the case of angiolipoma, hyperintensity on T1-WI can be observed owing to its high fat content ${ }^{1,414)}$.

Cavernous hemangiomas have a tendency to bleed and grow, so early surgical excision is recommended as soon as possible ${ }^{14)}$. However, they are hypervascular lesions, so massive bleeding can be seen during the operation. En-bloc resection after coagulation is preferred ${ }^{4,6,14)}$. As in our case, most patients show a good prognosis and an improvement in their symptoms after surgical resection of a cavernous hemangioma ${ }^{1,4,7,10,12)}$.

\section{CONCLUSION}

Pure spinal epidural cavernous hemangioma is very rare. 
It can cause radiculopathy like intervertebral disc herniation, which makes the condition difficult to diagnosis preoperatively. MRI is a useful tool in helping to differentiate it from other diseases, and early surgical treatment is important.

\section{REFERENCES}

1. Bayri Y, Ekşi MŞ, Yalçınkaya Koç D, Konya D: Spinal epidural cavernous angioma: two case reports and review of the literature. Acta Orthop Traumatol Turc 49:459-464, 2015

2. Caruso G, Galarza M, Borghesi I, Pozzati E, Vitale M: Acute presentation of spinal epidural cavernous angiomas: case report. Neurosurgery 60:E575-E576, 2007

3. Floeth F, Riemenschneider M, Herdmann J: Intralesional hemorrhage and thrombosis without rupture in a pure spinal epidural cavernous angioma: a rare cause of acute lumbal radiculopathy. Eur Spine J 19 Suppl 2:S193-S196, 2010

4. Hemalatha AL, Ravikumar T, Chamarthy NP, Puri K: A pure epidural spinal cavernous hemangioma - with an innocuous face but a perilous behaviour!! J Clin Diagn Res 7:1434-1435, 2013

5. Jang D, Kim C, Lee SJ, Ryu YJ, Kim J: Pure spinal epidural cavernous hemangioma with intralesional hemorrhage: a rare cause of thoracic myelopathy. Korean J Spine 11:85-88, 2014

6. Khalatbari MR, Abbassioun K, Amirjmshidi A: Solitary spinal epidural cavernous angioma: report of nine surgically treated cases and review of the literature. Eur Spine J 22:542-547, 2013

7. Li TY, Xu YL, Yang J, Wang J, Wang GH: Primary spinal epidural cavernous hemangioma: clinical features and surgical outcome in 14 cases. J Neurosurg Spine 22:39-46, 2015

8. Ozkal B, Yaldiz C, Yaman O, Ozdemir N, Dalbayrak S: Extraosseous, epidural cavernous hemangioma with back pain. Pol J Radiol 80:206-209, 2015

9. Petridis AK, Doukas A, Hugo HH, Barth H, Mehdorn HM: A rare case of extradural lumbar nerve root cavernoma. Eur Spine J 20 Suppl 2:S348-S349, 2011

10. Rodríguez-Boto G, Rivero-Garvía M, Gutiérrez-González R, Pérez-Zamarrón A, Vaquero J: Spinal epidural cavernous angiomas. Neurologia 29:443-445, 2014

11. Sağlam D, Sari A, Cansu A: Spinal epidural cavernous hemangioma: a rare site of involvement. Spine J 16:e251, 2016

12. Shivaprasad S, Shroff G, Campbell GA: Thoracic epidural cavernous hemangioma imaging and pathology. JAMA Neurol 70: 1196-1197, 2013

13. Tekkök IH, Akpinar G, Güngen Y: Extradural lumbosacral cavernous hemangioma. Eur Spine J 13:469-473, 2004

14. Yaldiz C, Asil K, Ceylan D, Erdem S: Thoracic extraosseous epidural cavernous hemangioma. J Korean Neurosurg Soc 57: 65-67, 2015

15. Yunoki M, Suzuki K, Uneda A, Yoshino K: A case of dumbbellshaped epidural cavernous angioma in the lumbar spine. Surg Neurol Int 6 Suppl 10:S309-S312, 2015 